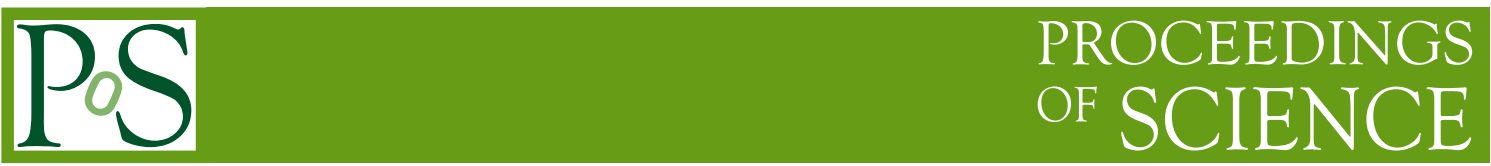

\title{
The First Light from MAXI
}

\section{S. Ueno ${ }^{* a}$ M. Matsuoka, ${ }^{a}$ K. Kawasaki, ${ }^{a}$ H. Tomida, ${ }^{a}$ M. Suzuki, ${ }^{a}$ M. Ishikawa, ${ }^{a}$ Y. Adachi, ${ }^{a}$ Y. Itamoto, ${ }^{a}$ T. Mihara, ${ }^{b}$ M. Kohama, ${ }^{b}$ M. Sugizaki, ${ }^{b}$ Y.E. Nakagawa, ${ }^{b}$ T. Yamamoto, ${ }^{b}$ H. Tsunemi, ${ }^{c}$ M. Kimura, ${ }^{c}$ N. Kawai, ${ }^{d}$ M. Morii, ${ }^{d}$ K. Sugimori, ${ }^{d}$} A. Yoshida,${ }^{e}$ K. Yamaoka, ${ }^{e}$ S. Nakahira, ${ }^{e}$ H. Negoro, ${ }^{f}$ R. Ishiwata, ${ }^{f}$ S. Miyoshi, ${ }^{f}$ H. Ozawa,${ }^{f}$ M. Nakajima, ${ }^{g}$ Y. Ueda,${ }^{h}$ N. Isobe, ${ }^{h}$ S. Eguchi, ${ }^{h}$ K. Hiroi, ${ }^{h}$ H. Katayayma ${ }^{i}$ and K. Ebisawa ${ }^{j}$

a ISS Science Project Office, ISAS, JAXA, 2-1-1 Sengen, Tsukuba, Ibaraki 305-8505, Japan

${ }^{b}$ Cosmic Radiation Laboratory, RIKEN, 2-1 Hirosawa, Wako, Saitama 351-0198, Japan

${ }^{c}$ Department of Earth and Space Science, Osaka University, Osaka 560-0043, Japan

${ }^{d}$ Department of Physics, Tokyo Institute of Technology, Tokyo 152-8551, Japan

${ }^{e}$ Department of Physics and Mathematics, Aoyama Gakuin University, 229-8558, Japan

${ }^{f}$ Department of Physics, Nihon University, Chiyoda-ku, Tokyo 101-8308, Japan

${ }^{g}$ School of Dentistry at Matsudo, Nihon University, Matsudo, Chiba 271-8587, Japan

${ }^{h}$ Department of Astronomy, Kyoto University, Oiwake-cho, Sakyo-ku, Kyoto 606-8502, Japan

${ }^{i}$ Earth Observation Research Center, JAXA, 2-1-1 Sengen, Tsukuba, Ibaraki 305-8505, Japan

${ }^{j}$ ISAS, JAXA, 3-1-1 Yoshinodai, Sagamihara, Kanagawa 229-8510, Japan

E-mail: ueno.shiro@jaxa.jp

Monitor of All Sky X-ray Image (MAXI) is the first astronomical payload on the International Space Station (ISS). MAXI was activated on 3 August 2009 by receiving electric power, circulated coolant, and data links from Japanese Experiment Module (JEM) "Kibo" Exposed Facility of ISS. All MAXI instruments have successfully passed the post-activation health check. MAXI has two types of X-ray cameras, GSC (Gas Slit Camera covering 2-30 keV with twelve proportional counters) and SSC (Solid state Slit Camera covering 0.5-12 keV with $32 \mathrm{X}$-ray CCD chips), and three support sensors, VSC (Visual Star Camera), RLG (Ring Laser Gyro), and GPSR (GPS Receiver). MAXI transfers telemetry data to the ground via data relay satellites. Having accumulated the GSC data for one ISS orbit (92 minutes), we released, on 18 August 2009, the "first light" image in which we can easily recognize about 20 bright Galactic sources. A preliminary analysis suggests that GSC achieved about 20-30 mCrab sensitivity in one orbit, mostly consistent with the pre-flight estimation. In January 2010, we started the public release of the MAXI light curves and images at http://maxi.riken.jp/ . We are preparing the automatic Internet transmission of the MAXI source detection alerts (MAXI Nova/Burst Alerts). The nominal mission life is five years.

The Extreme sky: Sampling the Universe above $10 \mathrm{keV}$ - extremesky2009,

October 13-17, 2009

Otranto (Lecce) Italy

\footnotetext{
* Speaker.
} 


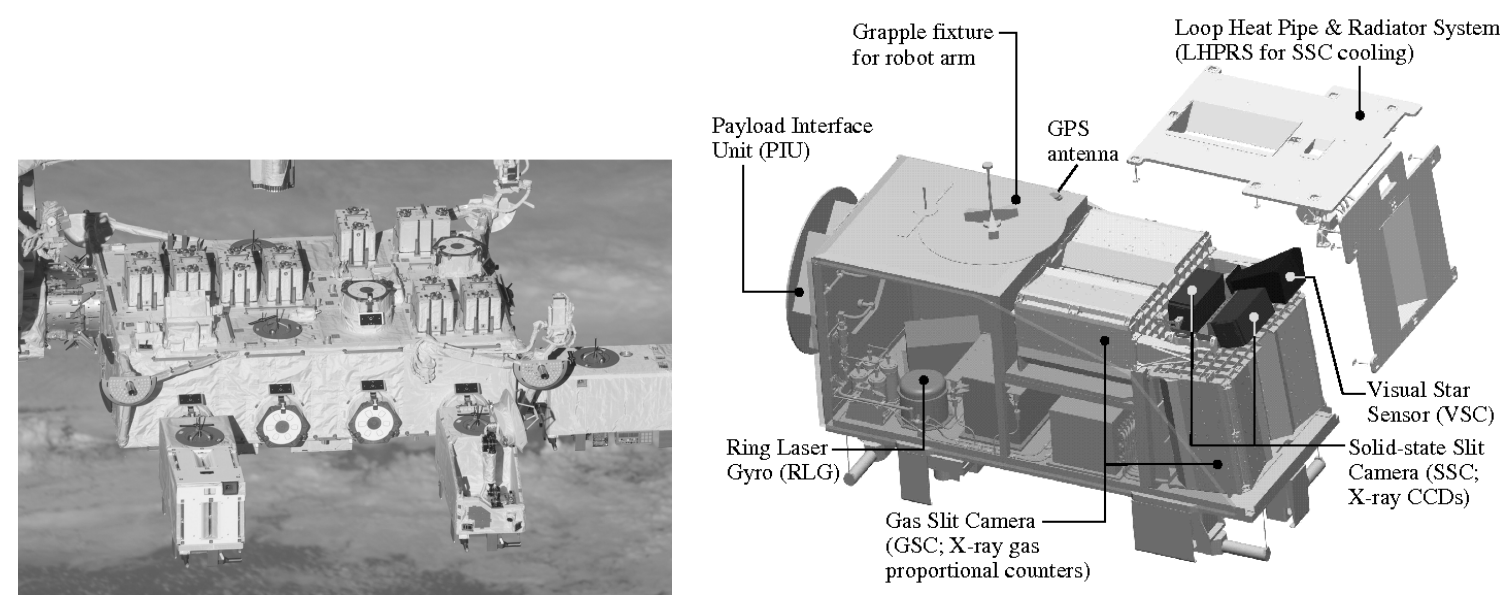

Figure 1: MAXI installed on the International Space Station.

Figure 2: MAXI payload overview. MAXI measures $185 \mathrm{~cm}$ long, $80 \mathrm{~cm}$ wide, and $100 \mathrm{~cm}$ high, and weighs $520 \mathrm{~kg}$.

\section{Introduction}

Monitor of All-sky X-ray Image (MAXI) is a 520-kg all-sky X-ray monitor, which were launched on Space Shuttle Endeavour at 7:03am JST on 16 July 2009, and installed on the International Space Station (ISS) at 00:24am JST on July 27 (see figure 1), and successfully activated on August 3. During the commissioning phase from August 2009 through March 2010, we evaluate the MAXI data to understand the instrument characteristics in orbit, the flight operations, and data processing. We started the public data release in January 2010.

The MAXI mission enables both monitoring and surveying the whole sky for five years or longer. MAXI alerts astronomers in case of GRB, X-ray novae, and any significant bright transient event. With the long-term data of X-ray sources, we can determine time scales of variability, e.g., long-term periodic or quasi-periodic motions of X-ray sources. MAXI will promote multiwavelength observations with other space and ground-based observatories in various bands, such as X-ray, infrared, optical, and radio. With MAXI's sensitivity, we can systematically investigate the variable activity of black hole binaries and AGNs. MAXI provides unbiased X-ray source catalogues over all the sky. Monthly or biannual X-ray catalogues could contribute to the longterm study of variable behavior of AGN as well as galactic X-ray sources.

MAXI is also able to make an all sky X-ray map with soft X-rays and medium energy X-rays. The soft X-ray map provides line features such as Ne and Oxygen $\mathrm{X}$-ray lines, which are useful in researching geo-coronal recombination lines as well as the evolution of hot gas in the Galaxy.

The MAXI science and instruments described before the launch have been presented in Matsuoka et al [1].

\section{Two Types of X-ray Cameras}

Figure 2 shows the MAXI overview and its subcomponents. MAXI has two types of X-ray cameras: Gas Slit Camera (GSC) covering 2-30 keV with twelve proportional counters; Solid 


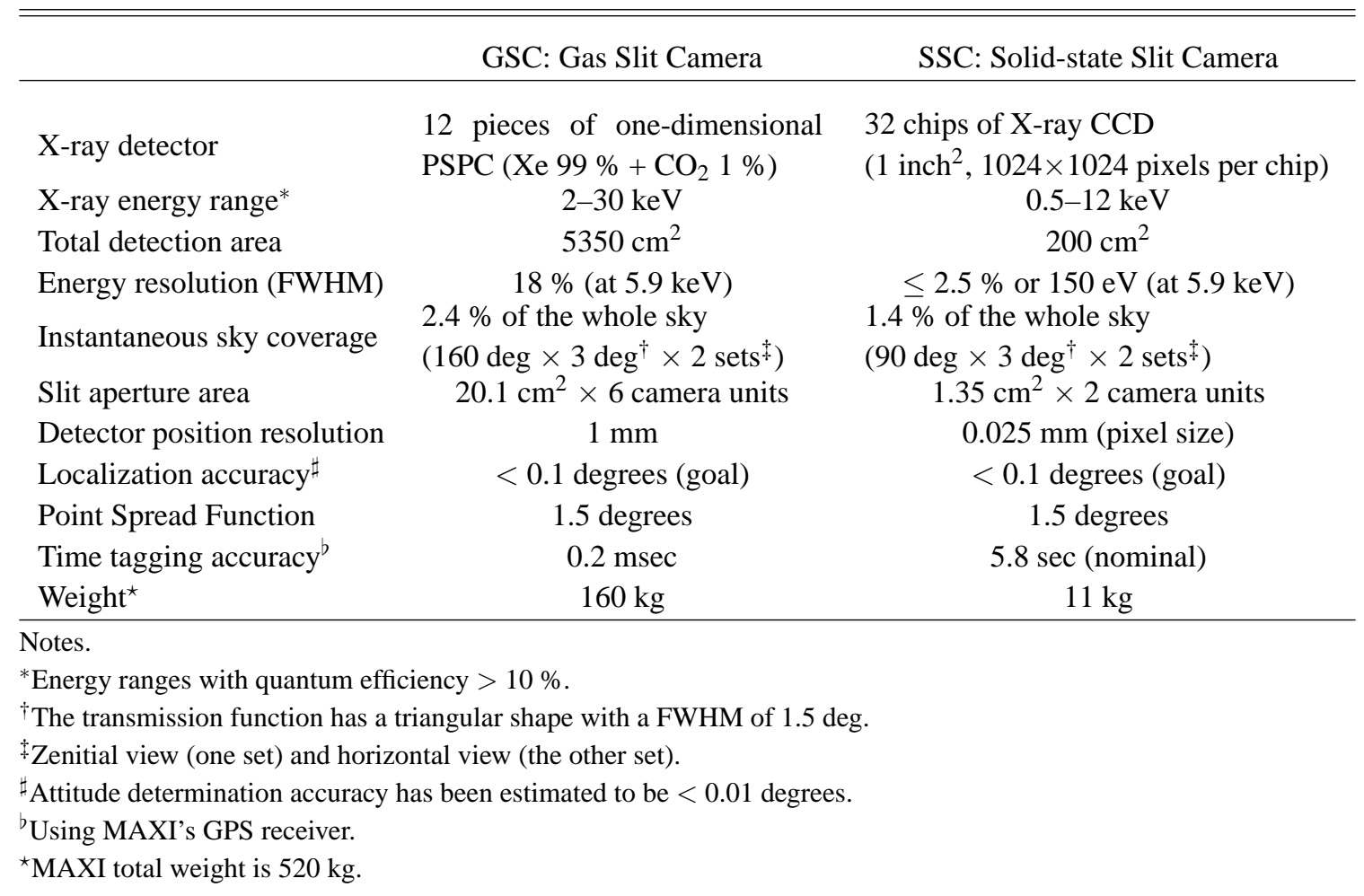

Table 1: Characteristics of the MAXI X-ray slit cameras.

State-slit Camera (SSC) covering 0.5-12 keV with $32 \mathrm{X}$-ray CCD chips. Table 1 lists characteristics of the MAXI X-ray cameras.

\subsection{Gas Slit Camera (GSC)}

GSC uses twelve pieces of one-dimensional position sensitive proportional counters (PSPC). Each set of two counters makes one camera unit, and hence GSC has six camera units in total. Three camera units view a horizontal direction from ISS; the other three, a zenithal direction (see figure 3). Each of the six GSC camera units has a field of view (FOV) of 80 degrees wide and 3 degrees across. The X-ray optics of each GSC camera unit is a combination of a slit and many parallelly aligned plates. The parallel plates over each PSPC limit its field of view to a three-degreewide area on a great circle of the sky, and the slit projects X-ray sources of different positions along the great circle onto different positions of the detector surface along its position sensitive direction. Adjacent GSC camera units have a 40-degree-wide overlap of their FOV (see figure 3), and therefore a horizontal (or zenithal) set of three camera units (right, center, and left) together makes a FOV of 160 degrees by 3 degrees. Since the line of sight of the "horizontal" camera units is tilted up towards the zenithal direction by 6 degrees, GSC does not see dark or bright Earth atmosphere as far as ISS stays within its allowable attitude range.

The two sets (horizontal and zenithal) of the GSC FOVs cover $2.4 \%$ of the whole sky, and hence MAXI is not optimized for the detections of short-duration X-ray brightenings. Nonetheless, we will issue the MAXI Nova/Burst alerts if we detect such short-duration events inside the MAXI 


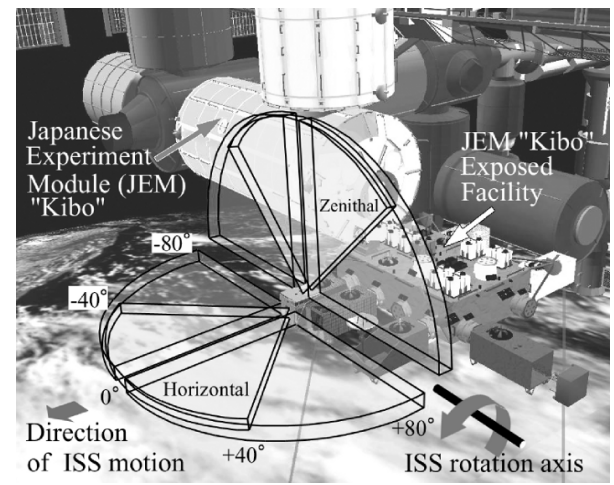

Figure 3: MAXI GSC fields of view.

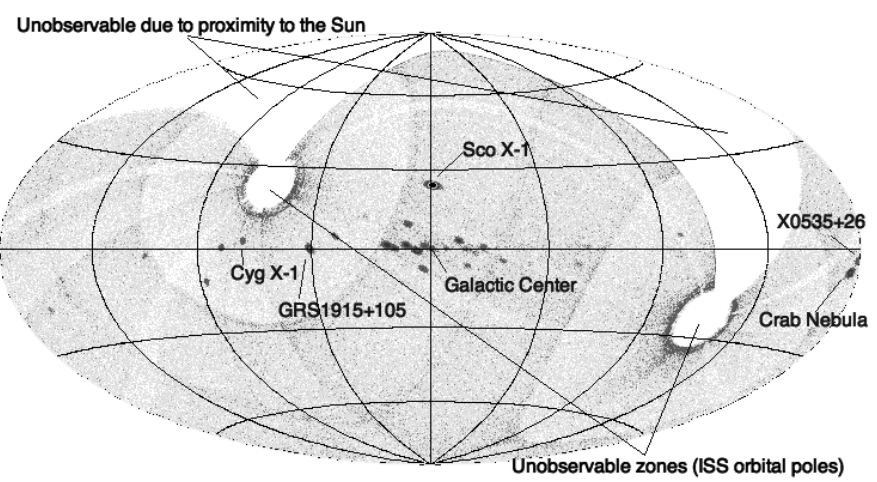

Figure 4: MAXI/GSC first light image.

FOV with good significance.

Going around the Earth, ISS always faces its one side toward the Earth center like an airplane. Every $\sim 92$ minutes when ISS completes one orbit, ISS also finishes one spin around the ISS rotation axis (see figure 3), and each of the horizontal and zenithal fields of view of GSC scans more than $90 \%$ of the whole sky. The sky region covered by the horizontal FOV is covered again by the zenithal field of view about 20 minutes later. Thus X-ray sources with variability time scales longer than several tens minutes will be good targets in the MAXI monitoring.

MAXI carries a GPS receiver, which enable us to attach a time tag to each GSC X-ray photon event with accuracy of $0.2 \mathrm{msec}$. Actual time scales which we can assess are limited by poor photon statistics due to MAXI's small slit apertures, but the time-tagging accuracy is useful when we study pulsation periods and their long-term changes by light curve folding.

\subsection{Solid-state Slit Camera (SSC)}

SSC consists of two camera units: one with a horizontal view; the other with a zenithal view. Each SSC camera unit uses an array of 16 chips laid out in a $2 \times 8$ format with no gaps in between. Each of the two SSC camera units has a field of view of 90 degrees wide and 3 degrees across. The $\mathrm{X}$-ray optics of each SSC camera unit is also a combination of a slit and many parallelly aligned plates. The line of sight of the "horizontal" camera unit is tilted up towards the zenithal direction by 16 degrees. The two sets (horizontal and zenithal) of the 90-degree wide SSC FOVs cover $1.4 \%$ of the whole sky. In one orbit, each of the horizontal and zenithal fields of view of SSC scans $\sim 70 \%$ of the whole sky.

SSC has a smaller slit aperture area and a smaller detection area than GSC, but SSC has higher soft X-ray sensitivity and higher energy resolution. SSC is essential to monitor soft X-ray sources and map the sky with soft X-ray lines.

\section{MAXI First Light Image}

On 18 August 2009, we released the MAXI first light image (figure 4), created from the GSC data accumulated for one ISS orbit from 3:00pm to 4:30pm JST of 15 August 2009. No exposure correction, no background subtraction, and no position correction has been applied. We can easily 


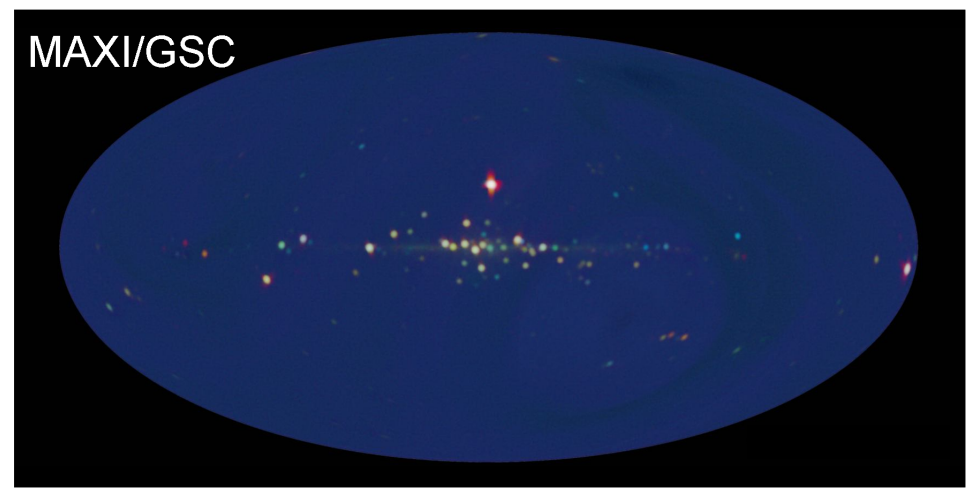

Figure 5: MAXI/GSC All-sky X-ray Image. No background subtraction or exposure correction is applied. Data accumulation time is about 2.5 months. Nearly $180 \mathrm{X}$-ray sources can be easily recognized.

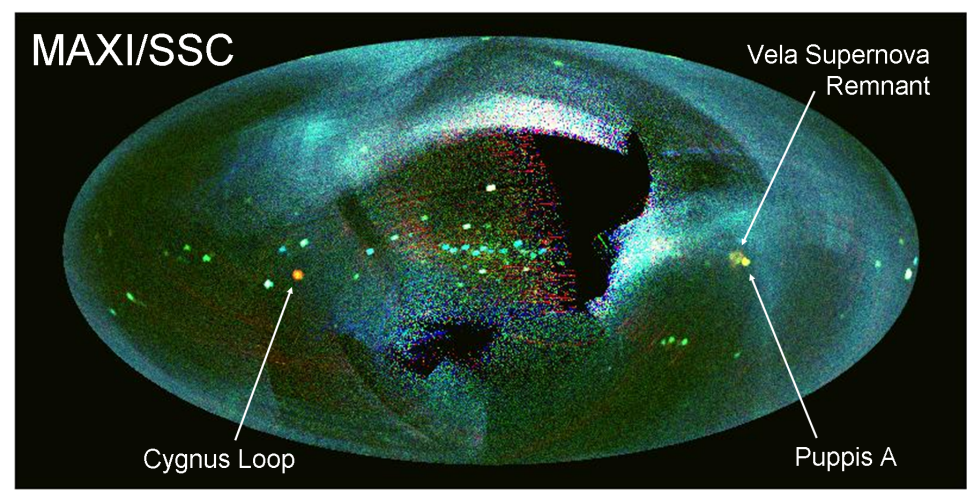

Figure 6: MAXI/SSC All-sky X-ray Image. No background subtraction or exposure correction is applied. Data accumulation time is nearly five months. To cover all the sky with SSC, we need six months.

recognize about 20 bright Galactic sources. A preliminary analysis of this first light image suggests that GSC achieved about 20-30 mCrab sensitivity in one orbit, mostly consistent with the pre-flight estimation of $5 \sigma$ detection limits: $20-25 \mathrm{mCrab}$ in the energy band of 2-30 keV for one ISS orbit (92 $\mathrm{min}$ ), 4-5 mCrab for one day, and $\sim 2 \mathrm{mCrab}$ for one week, reaching a source confusion limit of $\sim 0.2 \mathrm{mCrab}$ in every 1.5 years $[1]$.

The unobservable zone around the Sun is a result of the onboard sun avoidance angle set to 25 degrees. After the acquisition of the first light image, we have gradually reduced the sun avoidance angle of GSC to four degrees. Since ISS's orbital inclination is 51.6 degrees and its precession period is $\sim 2$ month, two disc-shaped unobservable areas (with radius of 10 degrees) around the ISS rotation poles will move along constant declination lines ( \pm 38.4 degrees) of the equatorial coordinate system, and become observable ten days later.

Figure 5 shows an all-sky image constructed from the GSC data obtained from 15 August to 29 October 2009. In this image, for which the background subtraction and the exposure correction have still not been applied, nearly 180 X-ray sources can be easily recognized. Figure 6 shows a nearly 5-month-accumulated SSC image. Three supernova remnants, Cygnus Loop, Vela Supernova Remnant, and Puppis A are recognized in the SSC all-sky image, but not in the GSC one, demonstrating the superior low-energy sensitivity of SSC. 


\begin{tabular}{|c|c|c|c|c|}
\hline MAXI Product* & Release Start & Latency & Contents & Status \\
\hline \multicolumn{5}{|l|}{ Nova/Burst Internet Alert ${ }^{\dagger}$} \\
\hline Manual transmission & August 2009 & $\sim 1$ day & position, time, brightness & Started \\
\hline Automatic transmission & Spring 2010 & $<30 \sec ^{b}$ & position, time, brightness & Planned \\
\hline \multicolumn{5}{|l|}{ Processed Data Files } \\
\hline Pre-selected sources ${ }^{\dagger}$ & January 2010 & $\sim 1$ day & light curve, image, spectrum ${ }^{\star}$ & Started \\
\hline Any sky regions $\sharp$ & September 2010 & $\sim 1$ day & light curve, image, spectrum ${ }^{\star}$ & Planned \\
\hline \multicolumn{5}{|l|}{ Notes. } \\
\hline \multicolumn{5}{|c|}{$\begin{array}{l}{ }^{*} \text { The web interface for the alert email registration and the processed data downloading is placed at http://maxi.riken.jp/ } \\
\text { †MAXI detections of significant transient events with various timescales will be reported through MAXI's alert ema } \\
\text { system and other existing routes, such as GCN(The Gamma-ray bursts Coordinates Network) and ATel(The As } \\
\text { tronomer's Telegram). }\end{array}$} \\
\hline \multirow{2}{*}{\multicolumn{5}{|c|}{$\begin{array}{l}\text { ¥At the beginning of the release, the number of pre-selected sources is } \sim 100 . \text { The number is increasing towards } \sim 1000 \\
\text { On the web interface, users can specify any time spans and sky regions. }\end{array}$}} \\
\hline & & & & \\
\hline \multicolumn{5}{|c|}{$\begin{array}{l}\text { Fastest case, using real-time downlinked data. A typical duration of real-time downlink is } 60-70 \% \text { of the total opera- } \\
\text { tion time. }\end{array}$} \\
\hline
\end{tabular}

Table 2: MAXI data products and public data release plan.

\section{Data Products and Public Release Plan}

Table 2 lists the MAXI data products and the release plan. We have already started the manual transmission of the nova/burst alerts. Before the manual transmission of each alert, the MAXI duty scientists carefully inspect and verify each transient event, and avoid false alerts. In this way we are actually able to inform the community not only about X-ray novae events but also about any other significant X-ray transient events detected in different timescales (from seconds to days). To receive the e-mail alerts is only necessary a mail list registration at http://maxi.riken.jp/ (figure 7). To inform the scientific community, we are also using the existing ways, such as ATels (The Astronomer' s Telegram) and GCN (The Gamma-ray bursts Coordinates Network). As of 15 January 2009, thirteen ATels (from [2] to [14]) and four GCN circulars (from [15] to [18]) have been already published.

We have not started yet the automatic Internet transmission of the MAXI source detection alerts (MAXI Nova/Burst Alerts). In fact we need further study beforehand to reduce the false detection rate of the MAXI Nova/Burst Alerts system by improving both the background subtraction and the source detection methods.

In January 2010, we started the public release of the MAXI light curves and images of about 100 pre-selected sources (figure 8). The number of the listed sources is increasing towards about 1000. The release of spectra will follow when we will be able to obtain background files and instrumental response files of acceptable quality. We will appreciate any suggestion for additional targets to the pre-selected list. The scientific community will be able to browse and download lightcurve, image and spectral files at http://maxi.riken.jp/ and analyze them with a software package, HEASoft/XANADU.

Figure 9 and Figure 10 show light curves and images of the black hole candidate XTE J1752-223 
[19] as an example of what will be possible to browse at and download from the MAXI public data release site.

In September 2010, we will open a web interface at http://maxi.riken.jp/, where it will be possible to specify any time span and sky region (not limited to the regions of the pre-selected sources) and download MAXI light curves, images and spectra of the selected sources.

Currently our scope of work does not include the public release of the low-level products, such as X-ray photon event data. By collaborating with the MAXI team, however, it is possible to access the MAXI low-level products for scientific analysis.

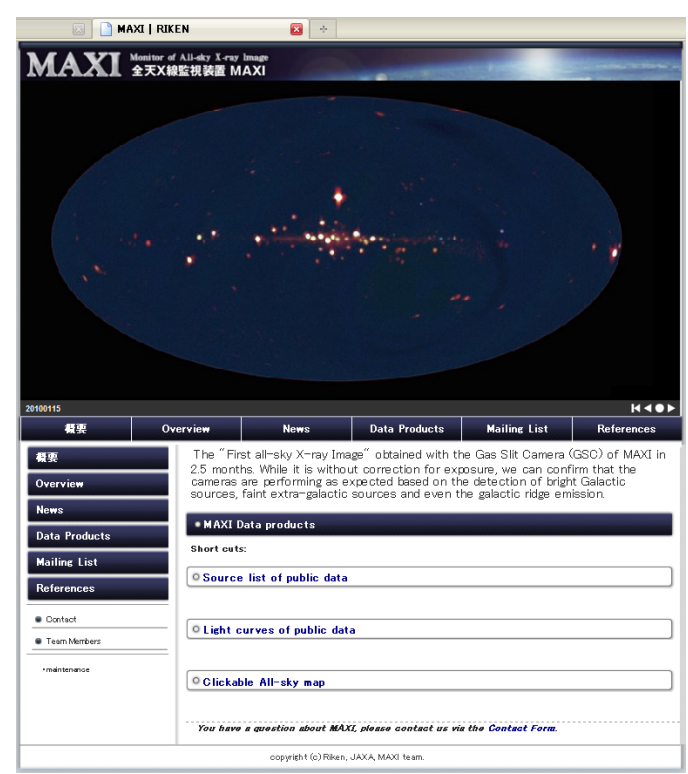

Figure 7: MAXI public data release site at http://maxi.riken.jp/, where you can browse and download the MAXI public released data, and can register to receive the MAXI email alerts.

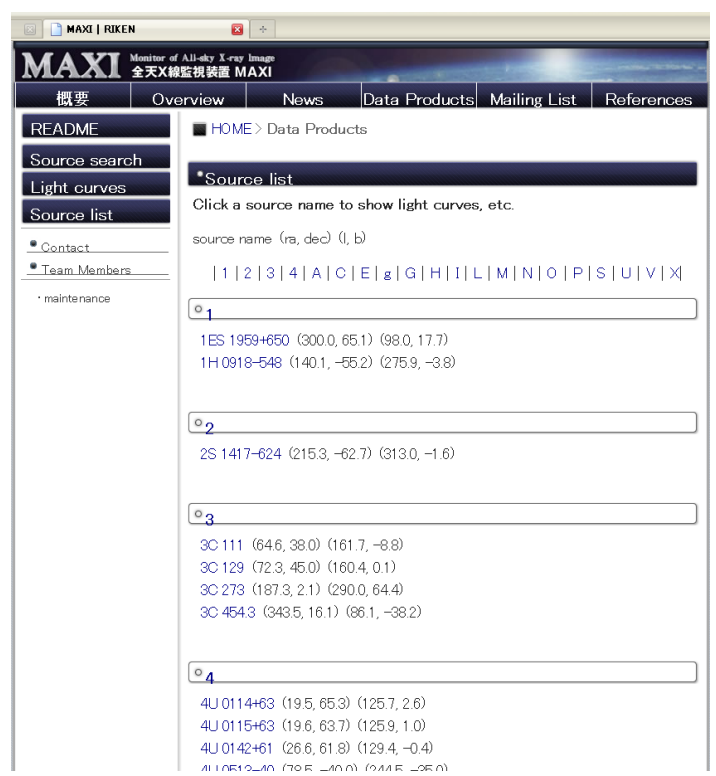

Figure 8: List of the sources selected for public data release. The number of the sources is currently about 100, increasing towards 1000 .

\section{References}

[1] M. Matsuoka, et al., The MAXI Mission of the ISS: Science and Instruments for Monitoring All-Sky X-Ray Images, PASJ 61 (2009) 999

[2] S. Nakahira, et al., XTE J1752-223: Flux increasing in a new RXTE and Swift X-ray transient in the Galactic center region, ATel \#2259 (2009)

[3] H. Negoro, et al., MAXI/GSC detection of an X-ray flare in the direction of $4 U 2206+54$, ATel \#2271 (2009)

[4] M. Sugizaki, et al., Renewed activity of A0535+26 detected by MAXI/GSC, ATel \#2277 (2009)

[5] T. Yamamoto, et al., MAXI/GSC detection of an outburst from GX 304-1, ATel \#2297 (2009)

[6] M. Nakajima, et al., Short X-ray Transient Detection by MAXI/GSC, ATel \#2321 (2009)

[7] H. Negoro, et al., MAXI/GSC detected an increasing soft X-ray component of Swift J1753.5-0127, ATel \#2341 (2009) 


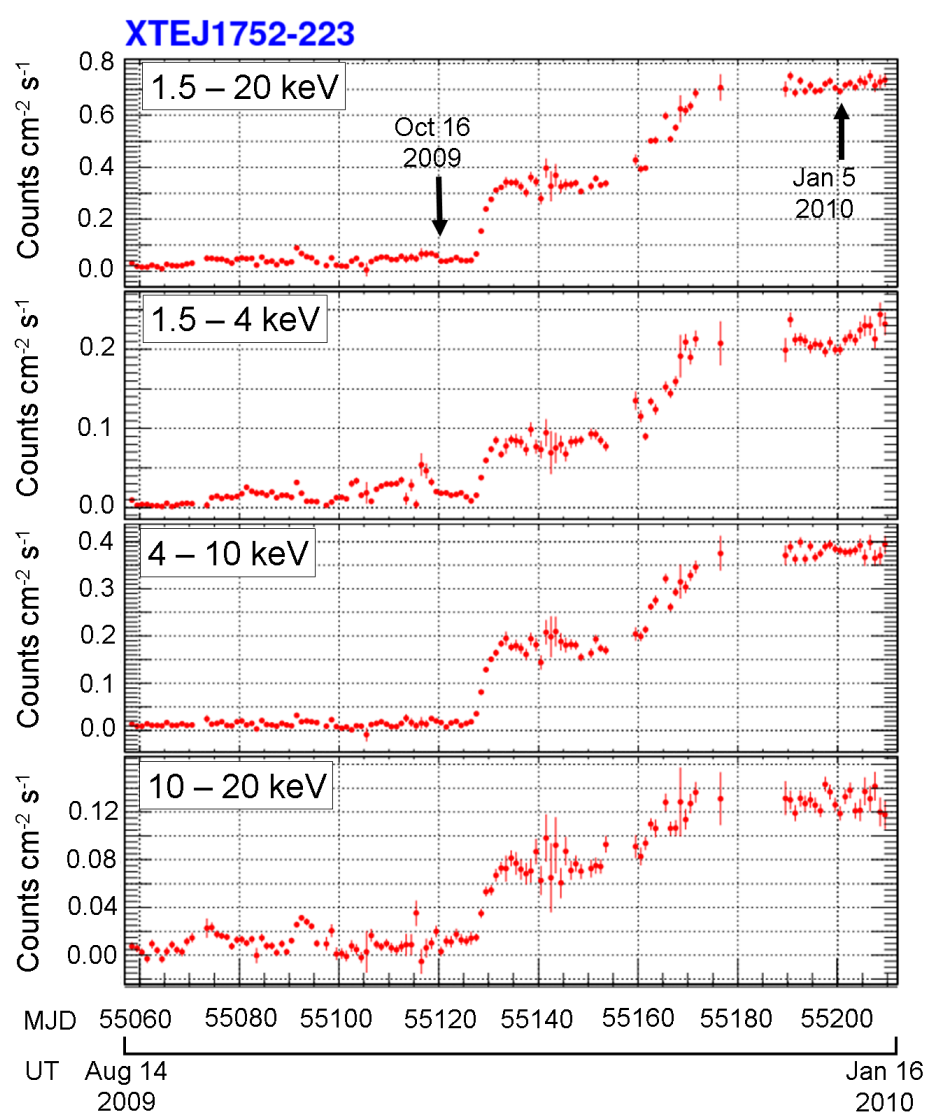

Oct 16, 2009 with MAXI/GSC (1-day observation)

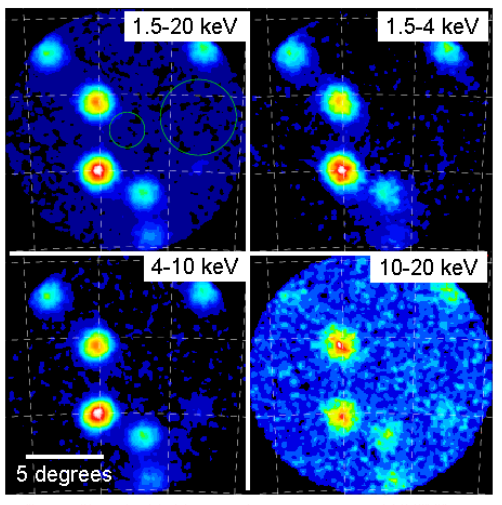

Jan 5, 2010 with MAXI/GSC (1-day observation)

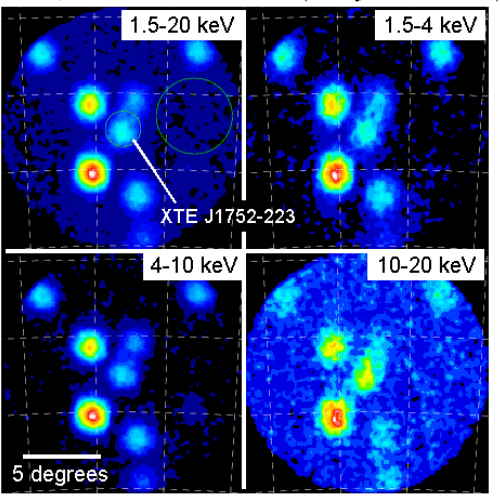

Figure 9: An example of the MAXI public released data (light

Figure 10: An example of the MAXI curves)

public released data (images)

[8] M. Suzuki, et al., MAXI/GSC detection of an X-ray outburst from NGC6440, ATel \#2360 (2009)

[9] H. Tomida, et al., MAXI/GSC detection of an increase in X-ray intensity from $4 U$ 1630-47, ATel \#2363 (2009)

[10] K. Yamaoka, et al., Renewed Activity from H 1743-322 detected by MAXI/GSC, ATel \#2364 (2009)

[11] N. Isobe, et al., MAXI/GSC detection of a bright X-ray flare from Mkn 421, ATel \#2368 (2010)

[12] S. Nakahira, et al., V0332+53: Renewed activity detected by MAXI/GSC, ATel \#2369 (2010)

[13] K. Yamaoka, et al., MAXI/GSC detects a spectral state transition in H 1743-322, ATel \#2378 (2010)

[14] K. Yamaoka, et al., MAXI/GSC detection of an X-ray activity from GX 339-4, ATel \#2380 (2010)

[15] M. Matsuoka, et al., GRB 090831: MAXI GSC detection, GCN Circular \#9852 (2009)

[16] M. Morii, et al., GRB 090926B: MAXI GSC detection, GCN Circular \#9943 (2009)

[17] M. Nakajima, et al., GRB 091120: MAXI GSC detection, GCN Circular \#10188 (2009)

[18] M. Nakajima, et al., Short X-ray Transient Detection by MAXI/GSC, GCN Circular \#10229 (2009)

[19] C. B. Markwardt, et al., XTE J1752-223: a new RXTE and Swift detected X-ray transient in the galactic center region, ATel \#2258 (2009) 